\title{
Speckle Noise Reduction from Medical Ultrasound Images-A comparative study
}

\author{
Ratil Hasnat Ashique ${ }^{1}$, Md. Imrul Kayes ${ }^{2}$ \\ ${ }^{1}$ Primeasia University Department of EEE ,12, Kamal Atartuk Avenue, Banani,Dhaka \\ ${ }^{2}$ International Islamic University Chittagong, Department of CSE, Chittagong,
}

\begin{abstract}
In medical image processing image denoising has become a very essential exercise all through the diagnose. Medical images are often deteriorated by noise due to a serious sources of interferences and other phenomenon that affect the measurement process in an imaging and acquisition system. Speckle noise is a random mottling of the image with bright and dark spots, which obscures fine details and degrades the delectability of low contrast lesions. Speckle noise occurrence is often undesirable since it affects the tasks of human interpretation and diagnosis. Speckle noise is a granular noise that inherently exists in and degrades the quality of the images. Generally it is found in Ultrasound image and radar image. Various noise removing methods has been introduced. This paper run a comparative study on various filter efficiencies in removing speckle noise from medical ultrasound images using statistical measures.
\end{abstract}

Keywords: Ultrasound Image,Ultrasonography, Speckle Noise, SNR, PSNR,MSE, RMSE

\section{Introduction}

Ultrasound image is formed from the sum of a large number of reflected waves, which scatters from the target. This scatters are randomly distributed and has random energy. This helps to form speckle noise. So this noise has a statistical nature which can be modeled. This section contains some primary knowledge about speckle noise and two Speckle noise's statistical model.

\section{Speckle Noise}

Speckle noise is a granular noise that inherently exists in and degrades the quality of the images. Generally it is found in Ultrasound image and radar image.

\section{Study and Modeling}

The study and modeling of this noise is important because speckle noise as the most prevalent artifact in Ultrasounds, makes object detection and recognition more difficult. Moreover reduction of speckle directly improves the value of a Sonogram.

\subsection{Basic Property}

i. Effects many types of Medical Imaging modalities Caused by constructive/destructive interference of random reflections.

ii. Random in nature. It Does not correspond to shape of object being imaged.

iii. It is deterministic. It does correspond to local density and ultrasound reflectivity.

iv. The Speckle Noise in Ultrasound is Multiplicative, not additive

v. Additive noise is considered insignificant compared to Speckle

\subsection{Consequences in Ultrasonography}

Consequences of ultrasonography can be described as follows:

i. Because Ultrasound Images have very little contrast, edge detection is essential to object detection.

ii. Ultrasounds depend more heavily on edge detection than other medical imaging modalities.

iii. Speckle noise can distort or hide edges making object detection less reliable.

iv. Objects such as tumors or birth defects can go undetected and thus untreated.

\section{Simulation}

The formation of speckle and the interference patterns in envelope image are caused by the coherent nature of echo ultrasound imaging systems. It will then reduce the image resolution and object detection 
ability. Although the speckle carries information related to the statistics of the scatters, various methods have been

proposed in the past involving frequency and spatial compounding to reduce the speckle. Here we will implement some of the filters, that descried in the previous chapter. The software used here is MATLAB. Among the implemented filters some are done by direct coding and some are done by image processing toolbox of MATLAB.

\section{Comparison Parameters}

The algorithms have been implemented using MATLAB. The performance of various spatial enhancement approaches are analyzed and discussed. The measurement of image enhancement is difficult to measure. There is no common algorithm for the enhancement of the image. The statistical measurement could be used to measure enhancement of the image. The Root Mean Square Error (RMSE), Signal-to-Noise Ratio (SNR), and Peak Signal-to-Noise Ratio (PSNR) are $t$ the standard deviation of the image:

Where, $i=1,2,3, \ldots, N, b$ is the mean gray level value of the original image and bi is the gray level value of the surrounding region and $\mathrm{N}$ is the total number of pixel in the image. The RMSE, SNR, and PSNR are provided below.

Table : 1

\begin{tabular}{|l|l|}
\hline $\begin{array}{l}\text { Statistical } \\
\text { Measurement }\end{array}$ & Formula \\
\hline MSE & $\operatorname{sum}\left(\mathrm{f}(\mathrm{I}, \mathrm{j})-\mathrm{F}(\mathrm{I}, \mathrm{j})^{\wedge} 2\right) / \mathrm{MN}$ \\
\hline RMSE & $\operatorname{sqrt}\left(\operatorname{sum}\left(\mathrm{f}(\mathrm{I}, \mathrm{j})-\mathrm{F}(\mathrm{I}, \mathrm{j})^{\wedge} 2\right) / \mathrm{MN}\right)$ \\
\hline SNR & $10 * \log \left((\operatorname{sigma})^{\wedge} 2\right) /\left(\operatorname{sigma}(\mathrm{e})^{\wedge} 2\right)$ \\
\hline PSNR & $20 * \log 10(255 / \mathrm{RMSE})$ \\
\hline
\end{tabular}

Here, $f(\mathrm{i}, \mathrm{j})$ is original image, $F(i, j)$ is enhanced image, $\sigma 2$ is variance of original image and $\sigma \mathrm{e} 2$ is variance of enhanced image. The original ultrasound image and filtered images of the prostate obtained by various filtering techniques are shown in figure 4.1. If the value of RMSE is low and the values of SNR and PSNR are larger then the enhancement approach is better. The table 2 shows the performance analysis of the proposed approaches and existing approaches with the regard to ultrasound medical images for prostate. It was observed from the figure 4.2 that the proposed M3-Filter removes the speckle noise better than other enhancement approaches.

V. Comparison Table

Table : 2

\begin{tabular}{|l|l|l|l|l|}
\hline FILTER & MSE & \multicolumn{1}{|c|}{ RMSE } & \multicolumn{1}{|c|}{ SNR } & PSNR \\
\hline Min & $\mathbf{0 . 0 0 7 9}$ & $\mathbf{0 . 1 0 1 3}$ & 25.3248 & $\mathbf{6 8 . 0 2 2 3}$ \\
\hline Max & 162.808 & 12.7596 & 10.6469 & 26.014 \\
\hline Mean & 4.9336 & 2.2212 & $\mathbf{4 0 . 2 9 0 1}$ & 41.1992 \\
\hline h. mean & 10.1359 & 3.1837 & 21.3762 & 38.0722 \\
\hline g. mean & 2.5016 & 2.958 & 21.2277 & 38.8358 \\
\hline Midpoint & 4.7037 & 2.1688 & 39.5441 & 41.4064 \\
\hline b.midpoint & 175.5446 & 13.2493 & 5.5354 & 25.6869 \\
\hline Sd & 9.9314 & 3.1514 & 14.335 & 38.1607 \\
\hline Variance & 84.1189 & 9.1716 & 4.2938 & 28.8819 \\
\hline Gaussian & 131.792 & 11.4801 & 4.2767 & 26.9316 \\
\hline a. median & 134.0236 & 11.5769 & 4.0548 & 26.859 \\
\hline
\end{tabular}




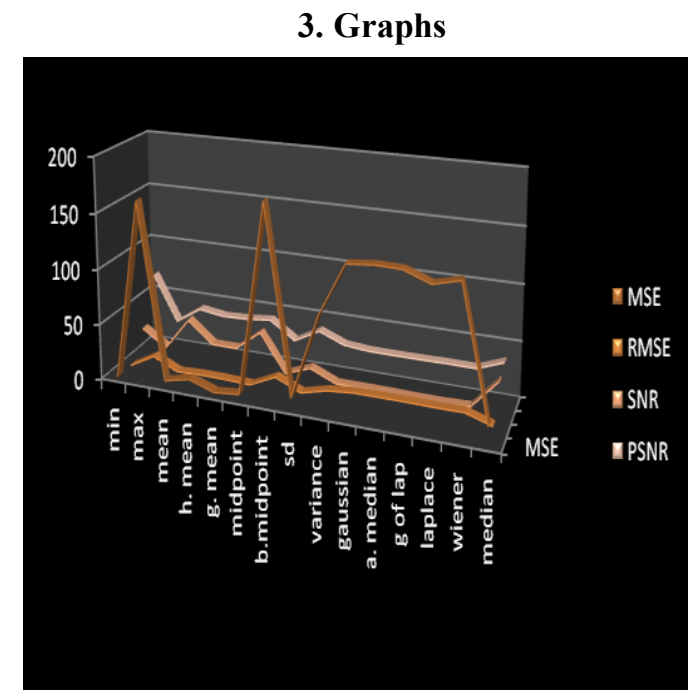

FIGURE: 1 Comparison of various parameters

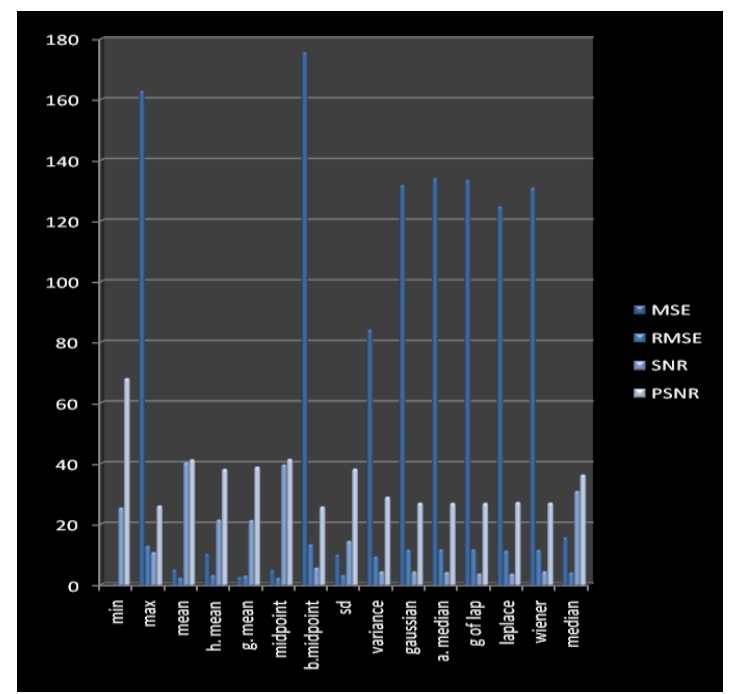

FIGURE: 2 bar comparison of various parameters

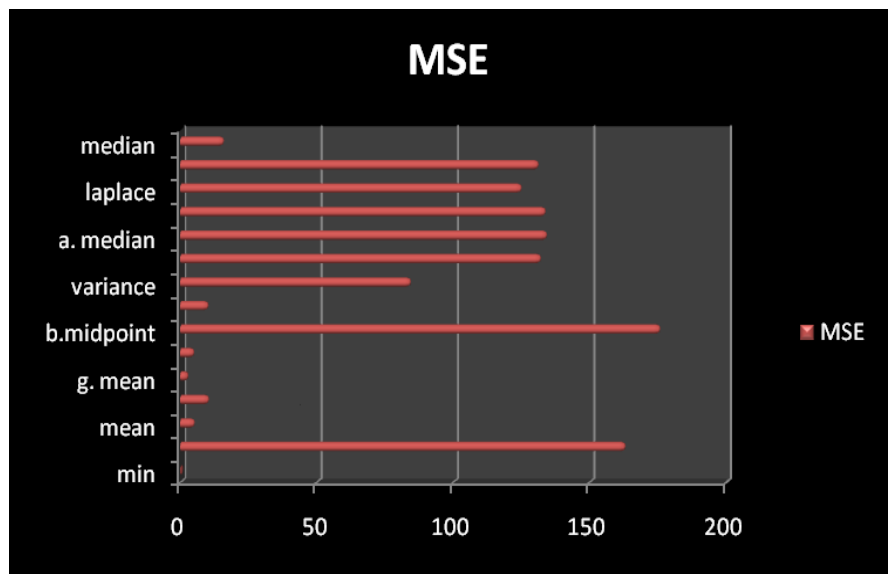

FIGURE : 3 MSE comparison 


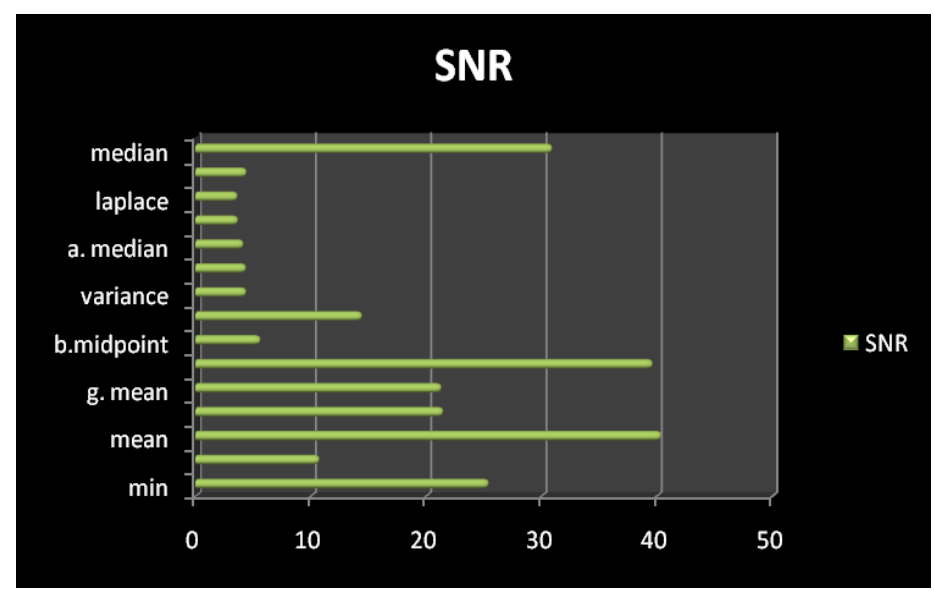

FIGURE: 4 SNR comparison

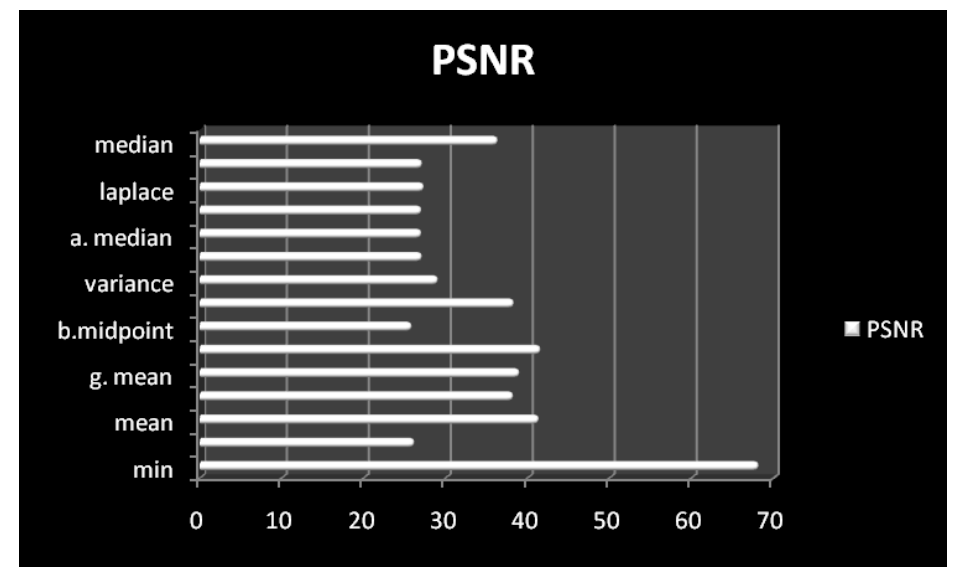

FIGURE : 5 PSNR comparison

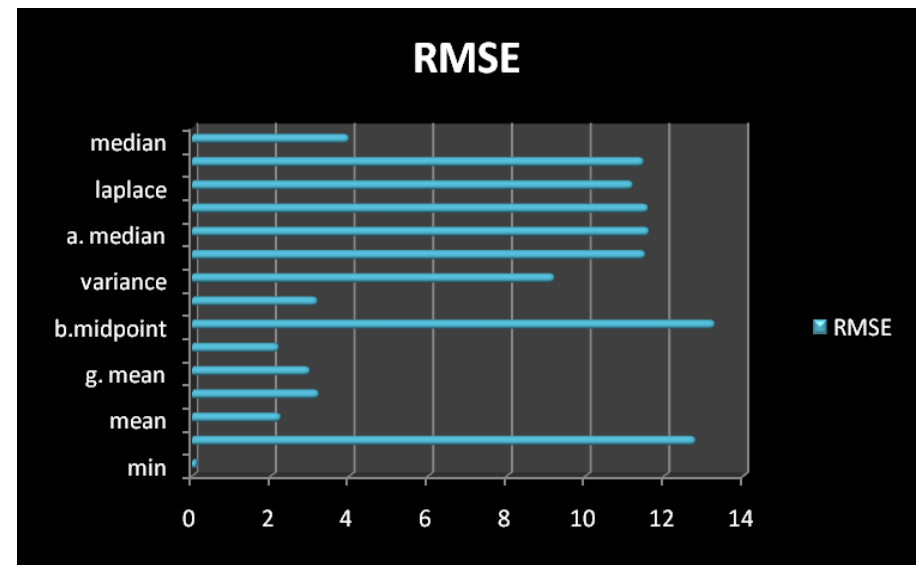

FIGURE : 6 RMSE comparison

\section{Observation}

According to the table -1 the min filter gives minimum MSE , minimum RMSE . Maximum SNR displayed by Mean filter. Moreover maximum PSNR is obtained from min filter. 


\section{References}

[1] Anil K.Jain, "Fundamentals of Digital Image Processing” first edition, 1989, Prentice - Hall, Inc.

[2] Tinku Acharya and Ajoy K. Ray, "Image Processing Principles and Appilications", 2005 edition A John Wiley \& Sons, Mc., Publication.

[3] Rafael C. Gonzalez and Richard E. Woods, "Digital Image Processing”, Second Edition, Pearson Education.

[4] "IEEE Computational Science and Engineering, summer" 1995, vol. 2, num.2, Published by the IEEE Computer Society, 10662 Los Vaqueros Circle, Los Alamitos, CA 90720, USA.

[5] Ingrid Daubechies "Ten lectures on wavelets" Philadelphia, PA: SLAM, 1992'

[6] Georges Oppenheim, "Wavelets and Their Application".

[7] S. Sudha, G.R Suresh and R. Suknesh, "Speckle Noise Reduction in Ultrasound images By Wavelet Thresholding Based On Weighted Variance", International Journal of Computer Theory and Engineering, Vol. 1, No. 1, PP 7-12,2009.

[8] S. Sudha, G.R Suresh and R. Suknesh, "Speckle Noise Reduction In ultrasound Images Using Context-Based Adaptive Wavelet Thresholding", IETE Journal of Research Vol 55 (issue 3), 2009.

[9] Zhenghao Shi and Ko B.Fung," A comparison of digital speckle filters" Canada centre for Remote Sensing.

[10] J.S.Lee,"Digital image enhancement and noise filtering by use of local Statistics"” IEEE Trans. Pattern Analysis and Machine Intelligence, vol.2,no. 2, pp. 165-168, March 1980.

[11] D.T.Kaun, A.A. Sawchuk, T.C. Strand, and P.Chavel,"Adaptive noise Smoothing filter for images with signal-dependent noise, ”IEEE Trans. Pattern Analysis and Machine Intelligence, vol.2,no. 2, pp. 165-177, March 1985.

[12] V.S.Frost, J.A Stiles, K.S. Shanmugan, and J.C. Holtzman, "A model for radar Images and its application to adaptive digital filtering of multiplicative noise," IEEE Trans. Pattern Analysis and Machine Intelligence, vol.2,no. 2, pp. 155-166 March 1980.

[13] Yongjian Yu and Scott T. Action, "Speckle Reducing Anisotropic Diffusion" IEEE Transaction on image processing, Vol. 11, NO. 11,pp. 1260-1270,NOV 2002.

[14] J.W. Goodman, Some fundamental properties of speckle, J.Opt. Soc. Am.,66: SS1145 - 1150, Nov 1976.[15]Yong Yue,Mihai M. Croitoru, Akhil Bidani, Joseph B. Zwischenberger and John W Clark,Jr.,'Ultrasound Speckle Suppression and Edge Enhancement Using multiscale nonlinear wavelet diffusion" ,IEEE $27^{\text {th }}$ Annual International Conference of the Engineering in medicine and biology society ,2005, page 6429-6432

[16] Chadsada Chinrungrueng and Aimamorn Suvichakron “Fast edge reduction for ultrasound images" IEEETRANSACTION ON NUCLEAR SCIENCE 2001,VOL 48 ,pages 849-854

[17] Yu Y, Acton S. Speckle reducing anisotropic diffusion. IEEE Trans Image Process 2002;11(11):1260-70.

[18] Yu, Y. Ultrasound image enhancement for detection of contours using speckle reducing anisotropic diffusion, PhD dissertation; May 2003.

[19] Speckle reducing anisotropic diffusion for 3D ultrasound images' Qingling Suna, John A. Hossackb,*, Jinshan Tangc, Scott T. Actonb,c Computerized Medical Imaging and Graphics 28 (2004) 461-470

Bibliography:

[20] Rafael C Gonzalez, Richard E. Woods (2002),DIGITAL IMAGE PROCESSING,2 ${ }^{\text {nd }}$ ed., Prentice Hall ,Upper saddle River,NJ 